\title{
Der Entwurf linearer Regelungssysteme im Zustandsraum*)
}

\author{
Von J. A CKERMANN, Oberpfaffenhofen
}

Es wird ein einfaches Verfahren zur Festlegung einer gewünschten charakteristischen Gleichung für ein System $\boldsymbol{x}[k+1]=$ $=\boldsymbol{A} \boldsymbol{x}[k]+\boldsymbol{b} u[k]$ durch eine Zustandsvektor-Rückführung $u=-\boldsymbol{k}^{\prime} \boldsymbol{x}$ hergeleitet, das keine Transformation der Zustandsgleichungen in eine kanonische Form erfordert. Das Ergebnis ist: Man erhält $\boldsymbol{k}^{\prime}$, indem man die gegebene Dynamik-Matrix $\boldsymbol{A}$ in das gewünschte charakteristische Polynomeinsetzt und mit der letzten Zeile der Inversen der Steuerbarkeitsmatrix vormultipliziert. Das duale Ergebnis für die Beobachter der Ordnung $n$ und der Ordnung $n-1$ wird gezeigt.

A simple method for obtaining a desired characteristic equation for a system $\boldsymbol{x}[k+1]=A \boldsymbol{x}[k]+\boldsymbol{b} u[k]$ by state variable feedback $u=-\boldsymbol{k}^{\prime} \boldsymbol{x}$ is derived. It does not require a transformation of the state equations to a canonical form. The result is: Obtain $\boldsymbol{k}^{\prime}$ by substituting the given dynamic matrix $\boldsymbol{A}$ into the desired characteristic polynomial and premultiplying by the last row of the inverse of the controllability matrix. The dual result for the observers of order $n$ and $n-1$ is shown.

\section{Einleitung}

Mit Hilfe von Zustandsraum-Methoden kann die günstigste Struktur eines linearen Regelungssystems gefunden werden. Es soll hier cin Verfahren hergeleitet werden, mit dem die freien Parameter in dieser Struktur auf einfache Weise so bestimmt werden können, daß das Regelungssystem eine gewünschte Dynamik erhält.

Der Übersichtlichkeit halber wird die Arbeit auf Systeme mit einer Eingangsgröße $u$ und einer Ausgangsgröße $y$ beschränkt. Außerdem werden hier nur diskrete Systeme behandelt; für kontinuierliche Systeme lassen sich alle Herleitungen völlig entsprechend durchführen, man muß im folgenden lediglich $f[k]$ durch $f(t), f[k+1]$ durch $\mathrm{d} f(t) / \mathrm{d} t$ usw. sowie $z$ durch $s$ ersetzen.

Die Regelstrecke sei durch die Zustandsgleichungen

$$
\begin{aligned}
\boldsymbol{x}[k+1] & =\boldsymbol{A} \boldsymbol{x}[k]+\boldsymbol{b} u[k], \\
y[k] & =\boldsymbol{c}^{\prime} \boldsymbol{x}[k]
\end{aligned}
$$

beschrieben, die Dimension der Dynamik-Matrix $\boldsymbol{A}$ sei $n . n$. Es wird vorausgesetzt, daß die Regelstrecke (1) steuerbar und beobachtbar ist, d. h. es sei

$$
\begin{aligned}
\operatorname{det} Q_{S} & =\operatorname{det}\left[\begin{array}{l}
\left.\boldsymbol{b}, \boldsymbol{A} \boldsymbol{b} \ldots \boldsymbol{A}^{n-1} \boldsymbol{b}\right] \neq 0, \\
\operatorname{det} \boldsymbol{Q}_{B}=\operatorname{det}\left[\begin{array}{l}
\boldsymbol{c}^{\prime} \\
\boldsymbol{c}^{\prime} \boldsymbol{A} \\
\vdots \\
\boldsymbol{c}^{\prime} \boldsymbol{A}^{n-1}
\end{array}\right] \neq 0 .
\end{array}\right.
\end{aligned}
$$

Im folgenden wird zunächst angenommen, der Zustand $\boldsymbol{x}$ sei bekannt. Durch eine Zustandsvektor-Rückführung kann damit eine gegebene charakteristische Gleichung erzeugt

Manuskripteingang: 17. August 1971.

*) Mitteilung aus dem DFVLR-Institut für Dynamik der Flugsysteme, 8031 Oberpfaffenhofen. werden. Das nicht meßbare $\boldsymbol{x}$ wird dann durch einen Schätzwert $\hat{x}$ ersetzt, den ein ,Beobachter" erzeugt, das ist ein dynamisches System mit den Eingangsgrößen $u$ und $y$.

\section{Zustandsvektor-Rückführung}

Durch eine Zustandsvektor-Rückführung

$$
u[k]=-\boldsymbol{k}^{\prime} \boldsymbol{x}[k]
$$

erhält das System (1) die Form

$$
\boldsymbol{x}[k+1]=\left(\boldsymbol{A}-\boldsymbol{b} \boldsymbol{k}^{\prime}\right) \boldsymbol{x}[k]=\boldsymbol{F} \boldsymbol{x}[k] .
$$

Der Parametervektor $\boldsymbol{k}$ soll so gewählt werden, daß das System (5) eine vorgegebene charakteristische Gleichung

$$
\begin{aligned}
R(z) & =\operatorname{det}(z \boldsymbol{I}-\boldsymbol{F})=\operatorname{det}\left(z \boldsymbol{I}-\boldsymbol{A}+\boldsymbol{b} \boldsymbol{k}^{\prime}\right)= \\
& =\alpha_{0}+\alpha_{1} z+\ldots+\alpha_{n-1} z^{n-1}+z^{n}=0
\end{aligned}
$$

erhält. Ein konstruktiver Beweis dafür, daß dies bei steuerbaren Systemen stets möglich ist, wurde von Bass und Gura [1] geführt. Ihr Beweis ist jedoch recht aufwendig, und im Ergebnis werden die Koeffizienten $a_{i}$ der charakteristischen Gleichung von $A$ benötigt. Eine einfache Beweisführung wird von Kalman [2] angegeben. Dabei werden die Zustandsgleichungen zunächst in die Regelungs-Normalform transformiert, das Ergebnis ist dann leicht abzulesen. Es müssen auch hierbei die $a_{i}$ berechnet werden. Wenn man in das ursprüngliche Koordinatensystem zurück will, muß außerdem die Transformationsmatrix invertiert werden. Für die praktische Anwendung ist daher auch dieser Weg nicht besonders vorteilhaft. Im folgenden wird eine neue Herleitung angegeben, die zu einem einfacheren Ergebnis führt. Der besondere Vorteil dieser Lösung ist, daß die $a_{i}$ nicht berechnet werden müssen.

Durch wiederholte Anwendung von Gl. (1) erhält man

$\boldsymbol{x}[k+1]=\boldsymbol{A} \boldsymbol{x}[k]+\boldsymbol{b} u[k]$,

$\boldsymbol{x}[k+2]=\boldsymbol{A}^{2} \boldsymbol{x}[k]+\boldsymbol{b} u[k+1]+\boldsymbol{A} \boldsymbol{b} u[k]$,

$\boldsymbol{x}[k+n]=\boldsymbol{A}^{n} \boldsymbol{x}[k]+\left[\boldsymbol{b}, \boldsymbol{A} \boldsymbol{b}, \ldots \boldsymbol{A}^{n-1} \boldsymbol{b}\right]\left[\begin{array}{l}u[k+n-1] \\ u[k+n-2] \\ \vdots \\ u[k]\end{array}\right]$.

Da das System nach Gl. (2) steuerbar ist, kann es aus jedem gegebenen Anfangszustand $\boldsymbol{x}[k]$ in jeden gewünschten Endzustand $\boldsymbol{x}[k+n]$ überführt werden, und zwar mit einer Stellfolge, die man durch Auflösung von Gl. (7) erhält:

$$
\left[\begin{array}{l}
u[k+n-1] \\
u[k+n-2] \\
\vdots \\
u[k]
\end{array}\right]=Q_{S}^{-1}\left(\boldsymbol{x}[k+n]-\boldsymbol{A}^{n} \boldsymbol{x}[k]\right) \text {. }
$$

Die Stellfolge bezeichnet die Stufenhöhen der treppenförmigen Stellgröße. 
Beim Einführen einer Rückführung will man im Gegensatz zur Steuerung zum Zeitpunkt $k$ noch nicht die gesamte Stellfolge vorausberechnen, sondern nur $u[k]$ bestimmen, d. h., es wird nur die letzte Zeile von Gl. (8) benötigt. Bezeichnet man die letzte Zeile von $Q_{S^{-1}}$ mit $\boldsymbol{e}^{\prime}$, so lautet die letzte Zeile von Gl. (8)

$$
u[k]=\boldsymbol{e}^{\prime}\left(\boldsymbol{x}[k+n]-\boldsymbol{A}^{n} \boldsymbol{x}[k]\right) .
$$

Darin wird nach Gl. (5) $\boldsymbol{x}[k+n]=\boldsymbol{F}^{n} \boldsymbol{x}[k]$ eingesetzt und $F^{n}$ mit Hilfe des Cayley-Hamilton-Theorems [3] ausgedrückt. Dieses Theorem besagt, daß jede $n . n$. Matrix ihre eigene charakteristische Gleichung erfüllt, mit Gl. (6) also

$$
R(\boldsymbol{F})=\alpha_{0} I+\alpha_{1} \boldsymbol{F}+\ldots+\alpha_{n-1} \boldsymbol{F}^{n-1}+\boldsymbol{F}^{n}=0 .
$$

Damit wird

$u[k]=-\boldsymbol{e}^{\prime}\left(\alpha_{0} \boldsymbol{I}+\alpha_{1} \boldsymbol{F}+\ldots+\alpha_{n-1} \boldsymbol{F}^{n-1}+\boldsymbol{A}^{n}\right) \boldsymbol{x}[k]$.

Die letzte Zeile von $\boldsymbol{Q}_{S}{ }^{-1} \boldsymbol{Q}_{S}=\boldsymbol{I}$ lautet

$$
e^{\prime}\left[b, A b \ldots A^{n-1} b\right]=\left[\begin{array}{llll}
0 & . & 0 & 1
\end{array}\right] .
$$

In Gl. (11) ist also

$$
\begin{aligned}
e^{\prime} F & =e^{\prime}\left(A-b k^{\prime}\right)=e^{\prime} A, \\
e^{\prime} F^{2} & =e^{\prime} A\left(A-b k^{\prime}\right)=e^{\prime} A^{2}, \\
& \vdots \\
e^{\prime} F^{n-1} & =e^{\prime} A^{n-1} .
\end{aligned}
$$

Das Regelgesetz erhält damit die Form

$u[k]=-e^{\prime}\left(\alpha_{0} I+\alpha_{1} A+\ldots+\alpha_{n-1} A^{n-1}+A^{n}\right) x[k]$,

und mit der Schreibweise von Gl. (6) ist

das heißt

$$
u[k]=-\boldsymbol{e}^{\prime} R(\boldsymbol{A}) \boldsymbol{x}[k]=-\boldsymbol{k}^{\prime} \boldsymbol{x}[k],
$$

$$
\boldsymbol{k}^{\prime}=\boldsymbol{e}^{\prime} R(A) .
$$

In Worten: Man erhält den Parametervektor $\boldsymbol{k}^{\prime}$ des Regelgesetzes (4), indem man die Dynamik-Matrix $A$ in das gewünschte charakteristische Polynom $R$ nach Gl. (6) einsetzt und mit der letzten Zeile der Inversen der Steuerbarkeitsmatrix vormultipliziert.

\section{Zustandsschätzung durch einen Beobachter der Ordnung n}

Da der Zustand $\boldsymbol{x}$ im allgemeinen nicht meßbar ist, wird $\boldsymbol{x}[k]$ in dem Regelgesetz (4) durch einen Schätzwert $\hat{\boldsymbol{x}}[k]$ ersetzt. Luenberger [4] hat zu diesem Zweck einen Beobachter entwickelt: Ein lineares dynamisches System der Ordnung $n$ mit den Eingangsgrößen $u$ und $y$, allgemein also

$$
\hat{\boldsymbol{x}}[k+1]=\boldsymbol{F} \hat{\boldsymbol{x}}[k]+\boldsymbol{g} u[k]+\boldsymbol{h} y[k] .
$$

Der Zustand $\hat{x}$ des Beobachters soll möglichst genau gleich dem Zustand $\boldsymbol{x}$ der Regelstrecke (1) werden. Wir führen den Schätzfehler

$$
\tilde{x}=x-\hat{x}
$$

ein und erhalten durch Subtraktion der Gl. (16) von Gl. (1)

$$
\tilde{\boldsymbol{x}}[k+1]=\boldsymbol{F} \tilde{\boldsymbol{x}}[k]+\left(A-\boldsymbol{F}-\boldsymbol{h} \boldsymbol{c}^{\prime}\right) \boldsymbol{x}[k]+(\boldsymbol{b}-\boldsymbol{g}) u[k] .
$$

Die Ruhelage der Fehlerdynamik (18) soll

$$
\lim _{k \rightarrow \infty} \tilde{x}[k]=0
$$

sein. Das wird erreicht mit

$$
\boldsymbol{F}=\boldsymbol{A}-\boldsymbol{h} \boldsymbol{c}^{\prime} \text { und } \boldsymbol{g}=\boldsymbol{b} \text {. }
$$

Außerdem muß das so entstehende System

$$
\tilde{\boldsymbol{x}}[k+1]=\boldsymbol{F} \tilde{\boldsymbol{x}}[k]=\left(\boldsymbol{A}-\boldsymbol{h} \boldsymbol{c}^{\prime}\right) \tilde{\boldsymbol{x}}[k]
$$

stabil sein. Der Beobachter (16) erhält die Form

$$
\begin{aligned}
\hat{\boldsymbol{x}}[k+1] & =\boldsymbol{F} \hat{\boldsymbol{x}}[k]+\boldsymbol{b} u[k]+\boldsymbol{h} y[k]= \\
& =\boldsymbol{A} \hat{\boldsymbol{x}}[k]+\boldsymbol{b} u[k]+\boldsymbol{h}\left(y[k]-\boldsymbol{c}^{\prime} \hat{\boldsymbol{x}}[k]\right)= \\
& =\boldsymbol{A} \hat{\boldsymbol{x}}[k]+\boldsymbol{b} u[k]+\boldsymbol{h} \boldsymbol{c}^{\prime} \tilde{\boldsymbol{x}}[k] .
\end{aligned}
$$

Der Beobachter ist also ein Modell der Regelstrecke, das durch den meßbaren Fehler $\boldsymbol{c}^{\prime} \tilde{\boldsymbol{x}}[k]$ angetrieben wird. Freie Parameter sind die $n$ Komponenten des Vektors $\boldsymbol{h}$. Sie können bei jedem beobachtbaren System so festgelegt werden, daß sich in Gl. (20) eine gewünschte Fehlerdynamik ergibt.

Die Bestimmung von $\boldsymbol{h}$ kann auf die Lösung (15) zurückgeführt werden. Das charakteristische Polynom der Fehlergleichung (20) lautet

$$
\begin{aligned}
B(z) & =\operatorname{det}(z \boldsymbol{I}-\boldsymbol{F})=\operatorname{det}\left(z \boldsymbol{I}-\boldsymbol{F}^{\prime}\right)=\operatorname{det}\left(z \boldsymbol{I}-\boldsymbol{A}^{\prime}+\boldsymbol{c} \boldsymbol{h}^{\prime}\right)= \\
& =\beta_{0}+\beta_{1} z+\ldots+\beta_{n-1} z^{n-1}+z^{n} .
\end{aligned}
$$

Für vorgegebene $\beta_{i}$ soll daraus $\boldsymbol{h}^{\prime}$ bestimmt werden, das ist das duale Problem zu Gl. (6). Es entsprechen einander $\boldsymbol{A}$ und $\boldsymbol{A}^{\prime}, \boldsymbol{b}$ und $\boldsymbol{c}, \boldsymbol{k}^{\prime}$ und $\boldsymbol{h}^{\prime}, \alpha_{i}$ und $\beta_{i}, R$ und $B$. Die duale Lösung ist also nach $\mathrm{Gl}$. (15)

$$
\begin{aligned}
\boldsymbol{h}^{\prime} & =\boldsymbol{f}^{\prime} B\left(\boldsymbol{A}^{\prime}\right)=\boldsymbol{f}^{\prime} B^{\prime}(\boldsymbol{A}), \\
\boldsymbol{h} & =B(\boldsymbol{A}) \boldsymbol{f} .
\end{aligned}
$$

Darin ist $\boldsymbol{f}^{\prime}$ die letzte Zeile der transponierten inversen Beobachtbarkeitsmatrix

$$
Q_{B}^{\prime-1}=\left[c, A^{\prime} c \ldots A^{\prime n-1} c\right]^{-1} ;
$$

$f$ ist folglich die letzte Spalte von $Q_{B}{ }^{-1}$. In Worten: Man erhält den Parametervektor $\boldsymbol{h}$ des Beobachters, indem man die Dynamik-Matrix $\boldsymbol{A}$ in das gewünschte charakteristische Polynom (22) einsetzt und mit der letzten Spalte der Inversen der Beobachtbarkeitsmatrix nachmultipliziert.

\section{Der Beobachter der Ordnung $n-1$}

Man kann die Zustandsgleichungen (1) stets so transformieren, daß die meßbare Ausgangsgröße $y$ eine Zustandsgröße ist. In dieser Form ist unmittelbar zu sehen, daß der Beobachter nur für die übrigen $n-1$ Zustandsgrößen Schätzwerte bilden muß. Luenberger [4] hat gezeigt, daß es für ein System $n$-ter Ordnung einen Beobachter der Ordnung $n-1$ gibt. Er benutzt zur Beweisführung die Diagonalform der Zustandsgleichungen. Kalman [2] transformiert das System zunächst in die Beobachter-Normalform und modifiziert diese dann durch eine weitere Transformation, so daß ein zu beobachtendes Teilsystem der Ordnung $n-1$ entsteht. Im folgenden wird ein anderer Weg in Anlehnung an die Arbeit von Kortüm [5] angegeben, bei dem keine Transformation in eine kanonische Form ausgeführt werden muß.

In der Zustandsgleichung (1) wird $\boldsymbol{x}$ in einen Vektor $\boldsymbol{x}^{*}$ der Dimension $n-1$ und in die verbleibende Komponente $x_{n}$ zerlegt: $\boldsymbol{x}^{\prime}=\left[x_{1}, x_{2} \ldots x_{n}\right]^{\prime}=\left[\boldsymbol{x}^{*^{\prime}}, x_{n}\right]^{\prime}$. Mit entsprechender Unterteilung von $\boldsymbol{A}, \boldsymbol{b}$ und $\boldsymbol{c}$ lautet Gl. (1) dann

$$
\begin{aligned}
{\left[\begin{array}{l}
\boldsymbol{x}^{*}[k+1] \\
x_{n}[k+1]
\end{array}\right] } & =\left[\begin{array}{ll}
\boldsymbol{A}_{11} & \boldsymbol{a}_{1 n} \\
\boldsymbol{a}_{n 1}^{\prime} & a_{n n}
\end{array}\right]\left[\begin{array}{l}
\boldsymbol{x}^{*}[k] \\
x_{n}[k]
\end{array}\right]+\left[\begin{array}{c}
\boldsymbol{b}^{*} \\
b_{n}
\end{array}\right] u[k], \\
y[k] & =\left[\begin{array}{ll}
\boldsymbol{c}^{* \prime} & c_{n}
\end{array}\right]\left[\begin{array}{l}
\boldsymbol{x}^{*}[k] \\
x_{n}[k]
\end{array}\right] .
\end{aligned}
$$


Dabei wird vorausgesetzt, daß $c_{n} \neq 0$, was bei einem beobachtbaren System gegebenenfalls durch Umnumerieren der Zustandsgrößen im Vektor $\boldsymbol{x}$ stets erreicht werden kann. Es wird zunächst $x_{n}$ aus Gl. (25) berechnet und in Gl. (24) eingesetzt. Das entspricht einer linearen Transformation

$\boldsymbol{w}=T_{1} \boldsymbol{x}=\left[\begin{array}{ll}\boldsymbol{I}_{n-1} & 0 \\ c^{* \prime} & c_{n}\end{array}\right] \boldsymbol{x}, \quad \boldsymbol{T}_{1}^{-1}=\left[\begin{array}{ll}\boldsymbol{I}_{n-1} & \mathbf{0} \\ -c^{* \prime} / c_{n} & 1 / c_{n}\end{array}\right]$.

Aus den Gln. (24) und (25) wird

$$
\begin{aligned}
\boldsymbol{w}[k+1] & =\boldsymbol{T}_{1} \boldsymbol{A} \boldsymbol{T}_{1}^{-1} \boldsymbol{w}[k]+\boldsymbol{T}_{1} \boldsymbol{b} u[k]= \\
& =\left[\begin{array}{ll}
\boldsymbol{P} & \boldsymbol{q} \\
\boldsymbol{r}^{\prime} & s
\end{array}\right] \boldsymbol{w}[k]+\left[\begin{array}{l}
\boldsymbol{b}^{*} \\
t
\end{array}\right] u[k] \\
y[k] & =\left[\begin{array}{ll}
\boldsymbol{c}^{* \prime} & c_{n}
\end{array}\right] \boldsymbol{T}_{1}^{-1} \boldsymbol{w}[k]=\left[\begin{array}{lll}
0 . .0 & 1
\end{array}\right] \boldsymbol{w}[k] .
\end{aligned}
$$

Darin ist

$\boldsymbol{P}=\boldsymbol{A}_{11}-a_{1 n} c^{* \prime} / c_{n}, \quad q=a_{1 n} / c_{n}, \quad s=c^{* \prime} a_{1 n} / c_{n}+a_{n n}$,

$\boldsymbol{r}^{\prime}=c^{* \prime} \boldsymbol{A}_{11}+c_{n} \boldsymbol{a}_{n 1}^{\prime}-\left(\boldsymbol{c}^{* \prime} \boldsymbol{a}_{1 n}+c_{n} a_{n n}\right) c^{* \prime} / c_{n}$,

$t=\boldsymbol{c}^{* \prime} \boldsymbol{b}^{*}+c_{n} b_{n}$.

In einer zweiten Transformation

$\boldsymbol{v}=\left[\begin{array}{l}\boldsymbol{v}^{*} \\ v_{n}\end{array}\right]=T_{2} \boldsymbol{w}=\left[\begin{array}{lr}\boldsymbol{I}_{n-1} & -\boldsymbol{h} \\ \mathbf{0} & 1\end{array}\right] \boldsymbol{w}, \quad \boldsymbol{T}_{2}^{-1}=\left[\begin{array}{ll}\boldsymbol{I}_{n-1} & \boldsymbol{h} \\ \mathbf{0} & 1\end{array}\right]$

wird nun ein zunächst beliebiger Parametervektor $\boldsymbol{h}$ eingeführt. Man erhält aus Gl. (27)

$\boldsymbol{v}[k+1]=\boldsymbol{T}_{2} \boldsymbol{T}_{1} \boldsymbol{A} \boldsymbol{T}_{1}^{-1} \boldsymbol{T}_{2}^{-1} \boldsymbol{v}[k]+\boldsymbol{T}_{2} \boldsymbol{T}_{1} \boldsymbol{b} u[k]=$
$=\left[\begin{array}{ll}\boldsymbol{P}-\boldsymbol{h} \boldsymbol{r}^{\prime} & \boldsymbol{P} \boldsymbol{h}+\boldsymbol{q}-\boldsymbol{h} \boldsymbol{r}^{\prime} \boldsymbol{h}-\boldsymbol{h} \boldsymbol{s} \\ \boldsymbol{r}^{\prime} & \boldsymbol{r}^{\prime} \boldsymbol{h}+s\end{array}\right] \boldsymbol{v}[k]+\left[\begin{array}{c}\boldsymbol{b}^{*}-\boldsymbol{h} t \\ t\end{array}\right] u[k]$,

$y[k]=\left[\begin{array}{lll}0 \ldots & 0 & 1\end{array}\right] \boldsymbol{v}[k]=v_{n}[k]$.

In der Differenzengleichung wird $v_{n}[k]=y[k]$ eingesetzt und der Beobachter entsprechend Gl. (21) als Modell des Teilsystems der Ordnung $n-1$ aufgebaut:

$\hat{\boldsymbol{v}}^{*}[k+1]=\left(\boldsymbol{P}-\boldsymbol{h} \boldsymbol{r}^{\prime}\right) \hat{\boldsymbol{v}}^{*}[k]+\left(\boldsymbol{P} \boldsymbol{h}+\boldsymbol{q}-\boldsymbol{h} \boldsymbol{r}^{\prime} \boldsymbol{h}-\boldsymbol{h} s\right) y[k]+$

$$
+\left(\boldsymbol{b}^{*}-\boldsymbol{h} t\right) u[k] \text {. }
$$

Durch Subtraktion vom ersten Teilsystem in der Differenzengleichung (30) erhält man für den Schätzfehler $\tilde{\boldsymbol{v}}^{*}=\boldsymbol{v}^{*}-\hat{\boldsymbol{v}}^{*}$ die Beziehung

$$
\tilde{\boldsymbol{v}}^{*}[k+1]=\left(\boldsymbol{P}-\boldsymbol{h} \boldsymbol{r}^{\prime}\right) \tilde{\boldsymbol{v}}^{*}[k] .
$$

Durch die Wahl von $\boldsymbol{h}$ kann wieder in der in den Gln. (22) und (23) dargestellten Weise für die Fehlerdynamik jedes gewünschte charakteristische Polynom

$B(z)=\operatorname{det}\left(z \boldsymbol{I}-\boldsymbol{P}+\boldsymbol{h} \boldsymbol{r}^{\prime}\right)=\gamma_{0}+\gamma_{1} z+\ldots+\gamma_{n-2} z^{n-2}+z^{n-1}$

erzeugt werden; es ist

$$
\boldsymbol{h}=B(\boldsymbol{P}) \cdot\left[\begin{array}{l}
\boldsymbol{r}^{\prime} \\
\boldsymbol{r}^{\prime} \boldsymbol{P} \\
\vdots \\
r^{\prime} \boldsymbol{P}^{n-2}
\end{array}\right]^{-1}\left[\begin{array}{l}
0 \\
\vdots \\
0 \\
1
\end{array}\right] .
$$

In dem zu schätzenden Zustand $\hat{\boldsymbol{v}}$ erhält man die ersten $n-1$ Komponenten aus dem Beobachter (31), und die letzte ist nach Gl. (30) $v_{n}=y$. Im ursprünglichen Koordinatensystem lautet der Schätzwert

$$
\begin{aligned}
\hat{\boldsymbol{x}}=\boldsymbol{T}_{1}^{-1} \boldsymbol{T}_{2}^{-1} \hat{\boldsymbol{v}} & =\boldsymbol{T}_{1}^{-1} \boldsymbol{T}_{2}^{-1}\left[\begin{array}{l}
\hat{\boldsymbol{v}}^{*} \\
y
\end{array}\right]= \\
& =\left[\begin{array}{l}
\hat{\boldsymbol{v}}^{*}+\boldsymbol{h} y \\
\left(-c^{* \prime} \hat{\boldsymbol{v}}^{*}\right) / c_{n}+\left(1-c^{* \prime} \boldsymbol{h}\right) / c_{n}
\end{array}\right] .
\end{aligned}
$$

Diese Gleichung kann mit der Unterteilung des $\hat{x}$-Vektors entsprechend Gl. (24) etwas einfacher in der folgenden Form geschrieben werden:

$$
\begin{aligned}
& \hat{\boldsymbol{x}}^{*}=\hat{\boldsymbol{v}}^{*}+\boldsymbol{h} y, \\
& \hat{x}_{n}=\left(y-\boldsymbol{c}^{* \prime} \hat{\boldsymbol{x}}^{*}\right) / c_{n} .
\end{aligned}
$$

Man braucht also bei der Anwendung des Verfahrens die Transformationen $\boldsymbol{T}_{1}$ und $\boldsymbol{T}_{2}$ nicht auszurechnen. Man unterteilt vielmehr $A, b$ und $c$ in der in Gl. (24) und (25) angegebenen Weise und bestimmt daraus $\boldsymbol{P}, \boldsymbol{q}, \boldsymbol{r}, s$ und $t$ nach Gl. (28). Nach Gl. (33) legt man das gewünschte charakteristische Polynom fest und bestimmt daraus $\boldsymbol{h}$ über Gl. (34). Damit ist der Beobachter nach den Gln. (31) und (35) bestimmt.

\section{Das Regelungssystem}

Das Regelungssystem aus Regelstrecke, Beobachter und Zustandsvektor-Rückführung ist in Bild 1 dargestellt. Das

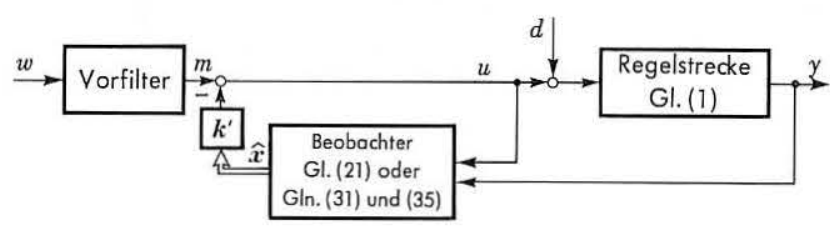

Bild 1. Regelungssystem mit zwei Freiheitsgraden.

Führungsverhalten von der Führungsgröße $w$ zur Regelgröße $y$ kann zusätzlich über ein Vorfilter beeinflußt werden, d. h. es wird eine Struktur mit zwei Freiheitsgraden [6] zugrunde gelegt. (Der Leser möge die von den deutschen Normen abweichende Bezeichnungsweise in Bild 1 entschuldigen. Dort wurde $y$ statt $x$ und in Gl. (1) $u$ statt $y$ benutzt, um mit den international gebräuchlichen Bezeichnungen in Übereinstimmung zu bleiben.)

Am Eingang der Regelstrecke wird eine nicht meßbare Störgröße $d$ angenommen. Damit lautet die vollständige Gleichung der Regelstrecke

$$
\begin{aligned}
\boldsymbol{x}[k+1] & =\boldsymbol{A} \boldsymbol{x}[k]+\boldsymbol{b}(u[k]+d[k]), \\
y[k] & =\boldsymbol{c}^{\prime} \boldsymbol{x}[k] .
\end{aligned}
$$

Mit

wird

$$
u=m-\boldsymbol{k}^{\prime} \hat{\boldsymbol{x}}=m-\boldsymbol{k}^{\prime} \boldsymbol{x}+\boldsymbol{k}^{\prime} \tilde{\boldsymbol{x}}
$$

$\boldsymbol{x}[k+1]=\left(\boldsymbol{A}-\boldsymbol{b} \boldsymbol{k}^{\prime}\right) \boldsymbol{x}[k]+\boldsymbol{b} \boldsymbol{k}^{\prime} \tilde{\boldsymbol{x}}[k]+\boldsymbol{b}(d[k]+m[k])$.

Verwendet man den Beobachter $n$-ter Ordnung nach Gl. (21), so erhält man entsprechend Gl. (20) den Schätzfehler $\tilde{\boldsymbol{x}}=\boldsymbol{x}-\hat{\boldsymbol{x}}$ durch Subtraktion der Gl. (21) von (36):

$$
\tilde{\boldsymbol{x}}[k+1]=\left(\boldsymbol{A}-\boldsymbol{h} \boldsymbol{c}^{\prime}\right) \tilde{\boldsymbol{x}}[k]+\boldsymbol{b} d[k] .
$$

In der Beschreibung des Gesamtsystems benutzt man wie in [2] den Schätzfehler $\tilde{\boldsymbol{x}}$ statt des Beobachterzustandes $\hat{\boldsymbol{x}}$. Die Gln. (38) und (39) werden also zusammengefaßt zu

$$
\begin{aligned}
{\left[\begin{array}{c}
\boldsymbol{x}[k+1] \\
\tilde{\boldsymbol{x}}[k+1]
\end{array}\right]=\left[\begin{array}{ll}
\boldsymbol{A}-\boldsymbol{b} \boldsymbol{k}^{\prime} & \boldsymbol{b} \boldsymbol{k}^{\prime} \\
0 & \boldsymbol{A}-\boldsymbol{h} \boldsymbol{c}^{\prime}
\end{array}\right]\left[\begin{array}{l}
\boldsymbol{x}[k] \\
\tilde{\boldsymbol{x}}[k]
\end{array}\right] } & +\left[\begin{array}{l}
\boldsymbol{b} \\
\boldsymbol{b}
\end{array}\right] d[k]+ \\
& +\left[\begin{array}{l}
\boldsymbol{b} \\
\mathbf{0}
\end{array}\right] m[k], \\
y[k]=\boldsymbol{c}^{\prime} \boldsymbol{x}[k] . &
\end{aligned}
$$


Die charakteristische Gleichung des Gesamtsystems lautet

$$
\begin{aligned}
& \operatorname{det}\left(z \boldsymbol{I}-\boldsymbol{A}+\boldsymbol{b} \boldsymbol{k}^{\prime}\right) \cdot \operatorname{det}\left(z \boldsymbol{I}-\boldsymbol{A}+\boldsymbol{h} \boldsymbol{c}^{\prime}\right)= \\
& =\left(\alpha_{0}+\ldots+\alpha_{n-1} z^{n-1}+z^{n}\right)\left(\beta_{0}+\ldots+\beta_{n-1} z^{n-1}+z^{n}\right)=0 .
\end{aligned}
$$

Hier treten also sowohl die für die Regelung in Gl. (6) festgelegten Eigenwerte also auch die Eigenwerte des Beobachters nach G1. (22) auf. Gl. (46) zeigt, daß der Beobachter und die Zustandsvektor-Rückführung sich nicht gegenseitig beeinflussen, sie liefert also nachträglich die Rechtfertigung für den Ersatz von $\boldsymbol{x}$ durch $\hat{\boldsymbol{x}}$.

Eine wichtige Eigenschaft dieses Regelungssystems ist, daß der Schätzfehler $\tilde{\boldsymbol{x}}$ von der Führungsgröße $m$ bzw. $w$ aus nicht steuerbar ist, d. h. das $\beta$-Polynom hat keinen Einfluß auf das Führungsverhalten. Man kannalso z. B. durchein Vorfilter $V(z)$ und die Wahl des $\alpha$-Polynoms die Führungs-Übertragungsfunktion $Y(z) / W(z)$ festlegen und danach die $\beta$-Koeffizienten im Hinblick auf eine günstige Störungsübertragungsfunktion $Y(z) / D(z)$ wählen. Wenn über die Störgröße $d$ zu wenig bekannt ist, können die Beobachterpole auch während des Betriebs nachgestellt werden, ohne daß sich das Führungsverhalten dabei ändert. Das gleiche gilt gemäß Gl. (32) auch für den Beobachter der Ordnung $n-1$.

\section{Ein Beispiel}

Die Regelstrecke in Gl. (1) sei gegeben durch

$$
\begin{aligned}
\boldsymbol{x}[k+1] & =\left[\begin{array}{cl}
2 & 1 \\
-0,5 & 0,5
\end{array}\right] \boldsymbol{x}[k]+\left[\begin{array}{l}
1 \\
0
\end{array}\right] u[k], \\
y[k] & =\left[\begin{array}{ll}
3 & 2
\end{array}\right] \boldsymbol{x}[k] .
\end{aligned}
$$

Die Steuerbarkeits-Matrix $Q_{S}$ und die Beobachtbarkeitsmatrix $\boldsymbol{Q}_{B}$ lauten

$$
\boldsymbol{Q}_{S}=[\boldsymbol{b}, \boldsymbol{A} \boldsymbol{b}]=\left[\begin{array}{cc}
1 & 2 \\
0 & -0,5
\end{array}\right], \quad \boldsymbol{Q}_{B}=\left[\begin{array}{l}
\boldsymbol{c}^{\prime} \\
\boldsymbol{c}^{\prime} \boldsymbol{A}
\end{array}\right]=\left[\begin{array}{ll}
3 & 2 \\
5 & 4
\end{array}\right] .
$$

Es ist $\operatorname{det} \boldsymbol{Q}_{S}=-0,5 \neq 0$ und $\operatorname{det} \boldsymbol{Q}_{B}=2 \neq 0$, das System ist also steuerbar und beobachtbar.

Es soll ein Regelungssystem mit $\alpha_{0}=\alpha_{1}=0$, d. h. $R(z)=z^{2}$ und einem Beobachter der Ordnung $n-1=1$ mit einem Pol bei $z=0,3$ entworfen werden.

Für die Zustandsvektor-Rückführung benötigen wir die letzte Zeile von $\boldsymbol{Q}_{S^{-1}}$. Sie lautet $\boldsymbol{e}^{\prime}=[0-2]$. Mit dem gewünschten charakteristischen Polynom $R(z)=z^{2}$ erhält man

$$
\boldsymbol{k}^{\prime}=\boldsymbol{e}^{\prime} R(\boldsymbol{A})=\boldsymbol{e}^{\prime} \boldsymbol{A}^{2}=\left[\begin{array}{ll}
2,5 & 0,5
\end{array}\right] .
$$

Beim Entwurf des Beobachters erster Ordnung können wir die Reihenfolge der $\boldsymbol{x}$-Komponenten beibehalten, da $c_{2}=2$ $\neq 0$. In Gl. (24) ist $A_{11}=2, a_{1 n}=1, a_{n 1}=-0,5, a_{n n}=0,5$, $b^{*}=1, b_{n}=0, c^{*}=3$ und $c_{n}=2$. Aus Gl. (28) erhält man damit $P=0,5, q=0,5, s=2, r=-1$ und $t=3$. Mit dem charakteristischen Polynom $-0,3+z$ nach Gl. (33) wird $h$ nach (34) $h=(-0,3+P)(-1)^{-1}=-0,2$. Die Beobachtergleichungen (31) und (35) lauten also

$$
\begin{aligned}
\hat{v}^{*}[k+1] & =0,3 \hat{v}^{*}[k]+0,84 y[k]+1,6 u[k], \\
\hat{x}^{*} & =\hat{v}^{*}-0,2 y, \\
\hat{x}_{2} & =\left(y-3 \hat{x}^{*}\right) / 2 .
\end{aligned}
$$

Das Regelgesetz $u=m-\boldsymbol{k}^{\prime} \hat{\boldsymbol{x}}$ erhält damit die Form

$u=m-\boldsymbol{k}^{\prime} \hat{\boldsymbol{x}}=m-\boldsymbol{k}^{\prime}\left[\begin{array}{c}\hat{x}^{*} \\ \hat{x}_{2}\end{array}\right]=m-1,75 \hat{v}^{*}+0,1 y$.

In dem Regelungssystem von Bild 1 ist der Beobachter mit der Zustandsvektor-Rückführung zusammengefaßt; sie werden zusammen durch die Gln. (43) und (44) beschrieben.

Es sei hier angemerkt, daß die Regelstrecke (42) instabil ist, wie man anhand der charakteristischen Gleichung $\operatorname{det}(z I-A)$ $=z^{2}=2,5 z+1,5=(z-1)(z-1,5)$ sieht. Die charakteristische Gleichung der Regelstrecke wird jedoch für das Entwurisverfahren nicht benötigt, und diẹ Instabilität der Regelstrecke ist ohne Bedeutung.

\section{Schlußbemerkungen}

Der Entwurf von Eingrößen-Regelungssystemen mit Beobachter und Zustandsvektor-Rückführung wurde vereinfacht. Nicht behandelt wurde dabei die Frage, wie die Regelungspole und Beobachterpole zu wählen sind. Diese Frage wird in [7] diskutiert. Dort wird auch die Erweiterung des Verfahrens auf Mehrgrößensysteme untersucht. Für den Fall, daß die gewünschte charakteristische Gleichung $z^{n}=0$ ist, wurde eine der Gl. (15) entsprechende Lösung für Mehrgrößensysteme, bereits in $[8, \mathrm{Gl} .(20)]$ angegeben

\section{Literatur}

[1] Bass, R. W. und Gura, I.: High order system design via state-space considerations. Preprints Joint Automatic Control Conference 1965, S. $311-318$

[2] Kalman, R. E., Falb, P. L. und Arbib, M. A.: Topics in mathematical system theory. McGraw Hill, New York 1969.

[3] Gantmacher, F. R.: Matrizenrechnung. VEB Deutscher Verlag der Wissenschaften, Berlin 1965.

[4] Luenberger, D. G.: Observing the state of a linear system. IEEE Trans. on Military Electronics 8 (1964) S. 74-80.

[5] Kortïm, W.: Zustandsschätzung durch Beobachter. Vortrag im Lehrgang Regelungstheorie der Carl-Cranz-Gesellschaft, Oberpfaffenhofen, Februar 1971.

[6] Horowitz, I. M.: Synthesis of feedback systems. Academic Press, New York 1963.

[7] Ackermann, J.: Abtastregelung. Springer Verlag, Berlin 1972.

[8] Ackermann, J.: Zeitoptimale Mehrfach-Abtastregelsysteme, Band I. Vorabdrucke zum IFAC-Symposium Mehrgrößen-Regelsysteme, Düsseldorf 1968 